\title{
Aluminum doped zinc oxide (AZO) coated optical fiber LMR refractometers - an experimental demonstration
}

\author{
Aritz Ozcariz ${ }^{1,2,}{ }^{,}$, Dafne A. Piña-Azamar ${ }^{3}$, Carlos R. Zamarreño ${ }^{1,2}$, René Dominguez ${ }^{3}$, Francisco \\ J. Arregui ${ }^{1,2}$ \\ ${ }^{1}$ Department of Electrical and Electronic Engineering, Public University of Navarre, 31006 Pamplona, Spain \\ ${ }^{2}$ Institute of Smart Cities, Public University of Navarre, 31006 Pamplona, Spain \\ ${ }^{3}$ Electrical and Electronics Engineering Department, UAM Reynosa-Rodhe, Universidad Autónoma de \\ Tamaulipas, Reynosa, Tamaulipas 88779, México. \\ *aritz.ozcariz@unavarra.es
}

\begin{abstract}
This work presents the experimental demonstration of lossy mode resonance generation by means of aluminum doped zinc oxide thin-films. The use of such material may allow to optimize the performance of LMR-based sensors, obtaining good sensitivity at lower costs than other overlays, such as those including indium. The refractometric response of the fabricated devices is explored in different spectral regions. One refractometer working in the near infrared region was fabricated, obtaining a sensitivity of 2,280 nm/RIU. A second refractometer working in the visible light spectrum was also fabricated. This second device allows to observe rough refractive index variations with the naked eye as a change of the color of the light propagating through the fiber, simplifying the setup needed for its use.
\end{abstract}

\section{Keywords}

Optical fiber; Refractometer; Optical Sensor; Lossy Mode Resonances (LMR); Aluminum Doped Zinc Oxide (AZO)

\section{Introduction}

Fiber optic sensors have gained wide importance over the last years [1-5]. The numerous benefits of optical fiber (low losses, electromagnetic noise immunity, small size, etc.) have facilitated the development of a big number of optical sensors based on several phenomena. Some of the most relatively recent sensors are those induced when a segment of optical fiber is coated with a thin-film that allows part of the wave to propagate through this film. Depending on the optical properties of this film, different kind of resonances can be generated, being the Surface Plasmon Resonances (SPR) and the Lossy Mode Resonances (LMR) the most widely studied. LMR are the focus of this research because of their wider spectral range and not being limited to a single polarization mode. For the generation of LMRs, the coating must have the real part of the permittivity positive, and its magnitude must be higher than both its own imaginary part and the permittivity of the media surrounding the coating [6].

Sensors based on Lossy Mode Resonances (LMR) have been widely studied in the last years. This technique has proven to provide high sensitivity and versatility in multiple applications such as refractometers, gas detectors, humidity sensors, etc. [1], obtaining astonishing figures of above 1 million nm/RIU in the silica refractive index region [7]. One of the most important factors in the performance of LMR based optical fiber sensors, is the supporting material coating the fiber. The fiber coating material optical properties define the characteristics of the sensors [8]. Therefore, it is of high importance to study the impact of the use of different materials. Transparent Conductive Oxide (TCO) [9-11] and polymers [12] are the main materials studied 
for this purpose. One example of them is aluminum doped zinc oxide (AZO). This material has optimal optical properties to generate LMR. In addition, AZO is non-toxic and it is widely available at lower cost than other materials employed for LMR generation such as ITO or tin oxide [13]. Doping zinc oxide with aluminum introduces several changes in the structure and the electrical and optical properties of the thin film $[14,15]$. Some simulations reported by other authors have pointed out the possible capability of AZO to be used as a coating material for the fabrication of LMR optical fiber sensors $[16,17]$. The economic and environmental benefits of the substitution of ITO thin films with other TCO such as AZO in the fabrication of electronical components has been addressed previously [18]. AZO has also been successfully used for the fabrication of electronical and SPR-based gas sensors (CO [19], formaldehyde [20], or sodium acetate [21], for example), although not for LMR-based optical fiber sensors. Considering all that, the introduction of AZO and other ZnO based films a step forward in the development of new sensors. To the best of our knowledge, this is the first report showing experimentally the generation of LMRs by means of sputtered AZO thin films.

\section{Materials and Methods}

Optical fiber sensors were obtained using a $4 \mathrm{~cm}$ segment of uncladded multimode optical fiber. This fiber was originally a FT200EMT plastic-clad 200/225 mm core/cladding diameter from Thorlabs Inc. The plastic cladding was chemically removed (burnt off) and then ultrasonically washed in detergent, deionized water, piranha solution and finally deionized water. The resulting fiber was then cleaved and spliced to optical fiber pigtails of the same dimensions at both ends. This device was placed into a sputtering chamber and coated with a thin film of AZO (Sputtering targets were obtained from Zhongnuo Advanced Materials Inc.). A pressure of $4 \cdot 10^{-}$ ${ }^{2}$ mbar of pure argon and $200 \mathrm{~mA}$ of current were used in the process. The absorption spectrum of the fiber was monitored during the deposition using the scheme shown in fig. 1 with the sensitive region placed in the sputtering chamber. The experimental setup (see fig. 1) included a halogen light source (from Spectral Products Inc.) and a set of two spectrometers (USB2000+XR1 and NIR512 from Ocean Optics Inc.), connected using a bifurcated fiber (VIS/NIR from Ocean Optics Inc.). During the tests, the sensitive region was submerged in several glycerin water solutions of increasing refractive index. Al the experiments were performed under the same conditions at room temperature $\left(25^{\circ} \mathrm{C}\right)$. An additional test was performed with solutions of indistinguishable RI variation with different $\mathrm{pH}$ (Panreac Inc. PBS buffer solutions) in the range of 6.58 to 7.67 to study the effect of $\mathrm{pH}$ variation in the measurements. 


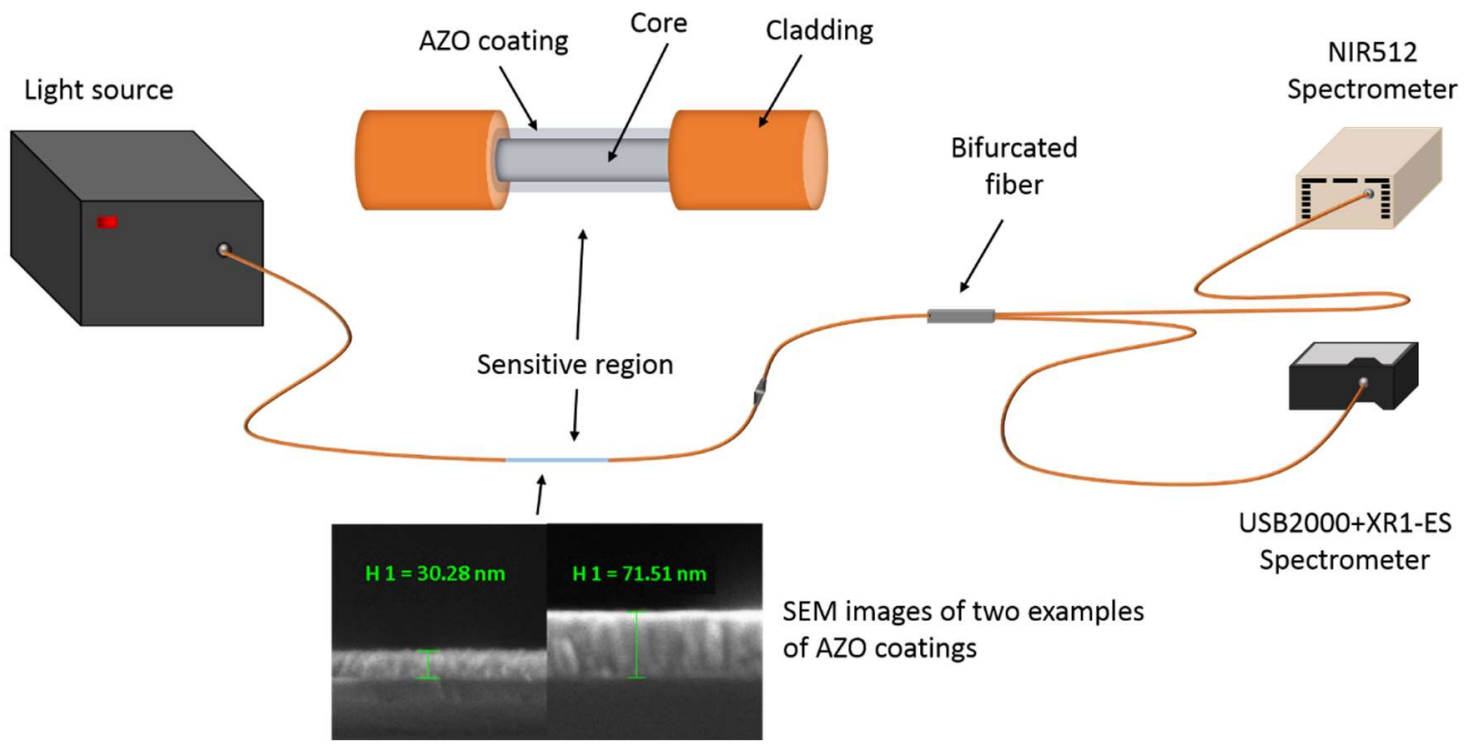

Figure 1: The experimental setup used in the experiments performed with the fabricated devices consisted of a halogen lamp connected to an optical fiber that propagates the light to the sensitive region, which is then connected to a bifurcated fiber that delivers the light to a set of two spectrometers. On the bottom of the figure we can see the detail of two SEM images obtained for AZO coatings of different thicknesses (30.28 nm and $71.51 \mathrm{~nm}$ ).

\section{Results and discussion}

Due to the novelty of the use of AZO as a supporting material for LMR generation, it is very interesting to study the absorption spectrum as a function of the coating thickness. Therefore, using the setup explained above, it is possible to observe the spectral response in real time during the AZO thin-film fabrication. These data are shown in fig. 2 . As the thickness of the film grows linearly during the sputtering deposition at an approximate rate of $12.9 \mathrm{~nm} / \mathrm{min}$, this graph also shows the evolution of the spectrum as the AZO film thickens. The coating fabricated on this fiber presents a thickness of $167 \mathrm{~nm}$. The color in this figure indicates the absorption value for a given wavelength at a particular time. It can be seen how several absorption peaks (resonances) appear at shorter wavelengths and shift to longer wavelengths as the thickness of the AZO film increases, which is the typical behavior of the LMR. It must be noted how the resonances of lower order (those that appear first, on the left in fig. 2), shift faster than the following resonances, which is also corroborated in previous studies with other materials [8]. Another aspect to consider is that these resonances seem to appear in couples. This effect can be explained taking into account that resonances have two components: one corresponding to a TE (Transverse Electric) mode of propagation, and another one corresponding to the TM (Transverse Magnetic) mode [22]. These two modes have sometimes their peak wavelength of resonance at slightly different wavelengths and each component can be observed separately even without the use of a polarizer. An example of this can be seen on fig. 2. In our setup there is no polarization separation and the sum of both components are seen at a time. Particularly, we can appreciate how the first LMR has two well differentiated components that seem to diverge as the film thickens. In the second LMR, its two components can still be observed, although they are closer. For the third and fourth order LMR, these two components cannot be differentiated with this setup. In order to study them individually, it would be necessary the use of a more complicated setup with polarization controllers and non-symmetrical optical fiber structures, such as D-Shape fibers. 


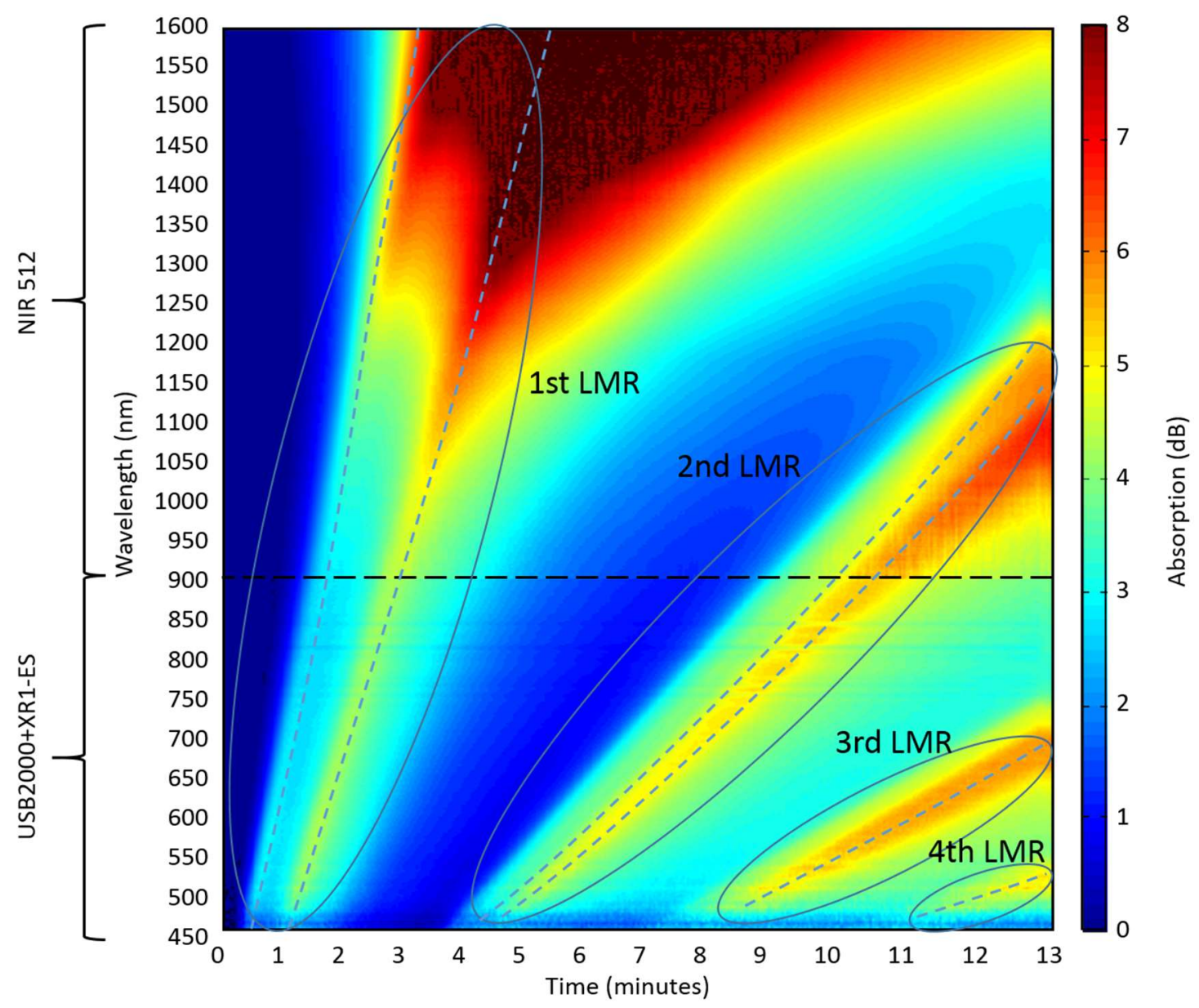

Figure 2: Absorption spectra in the VIS-NIR region for different times during the film deposition process. Several resonances can be observed, shifting to longer wavelengths as the film thickens.

Here it is important to remark that, since the sputtering process is performed in an argon atmosphere, the film may suffer a further oxidation once it comes in contact with oxygen. This effect was taken into account and studied. It was proven that, although this oxidation causes a red-shift (approx. $5 \mathrm{~nm}$ ) in the resonances, after a period of 8 hours at room temperature $\left(23^{\circ} \mathrm{C}\right)$ the coating is completely stabilized and the LMRs maintain their position.

Once the generation of resonances and their shift depending on the thickness of the coating has been experimentally demonstrated, it is interesting to study the sensitivity of such resonances to the surrounding medium refractive index (SMRI). The sensor fabricated in the process described above after 13 minutes deposition time (see fig. 2) shows a second order LMR on the NIR region (1090 $\mathrm{nm}$ ) and a third and fourth order LMR in the visible light spectrum (510 and $670 \mathrm{~nm}$ respectively). The first order LMR has already shifted out of the spectral working range and cannot be measured. In fact, the width of this first LMR associated to the separation of its components would make it hard to characterize the refractometric response using this simple setup, as it can be seen in the example spectrum shown in fig. $3 \mathrm{~b}$. Therefore, the response of the $2^{\text {nd }}, 3^{\text {rd }}$ and $4^{\text {th }}$ LMRs have been also studied as a function of the SMRI. The sensitive region was successively submerged in glycerin solutions of increasing refractive index while observing the absorption spectra. Fig. 3a shows the shift of each LMR to greater wavelengths as the refractive index increases. It can also be observed how this wavelength shift is greater in the $2^{\text {nd }}$ order LMR than in the $3^{\text {rd }}$ order LMR and the wavelength shift of the $3^{\text {rd }}$ order LMR is also greater than that of the $4^{\text {th }}$ order LMR. 

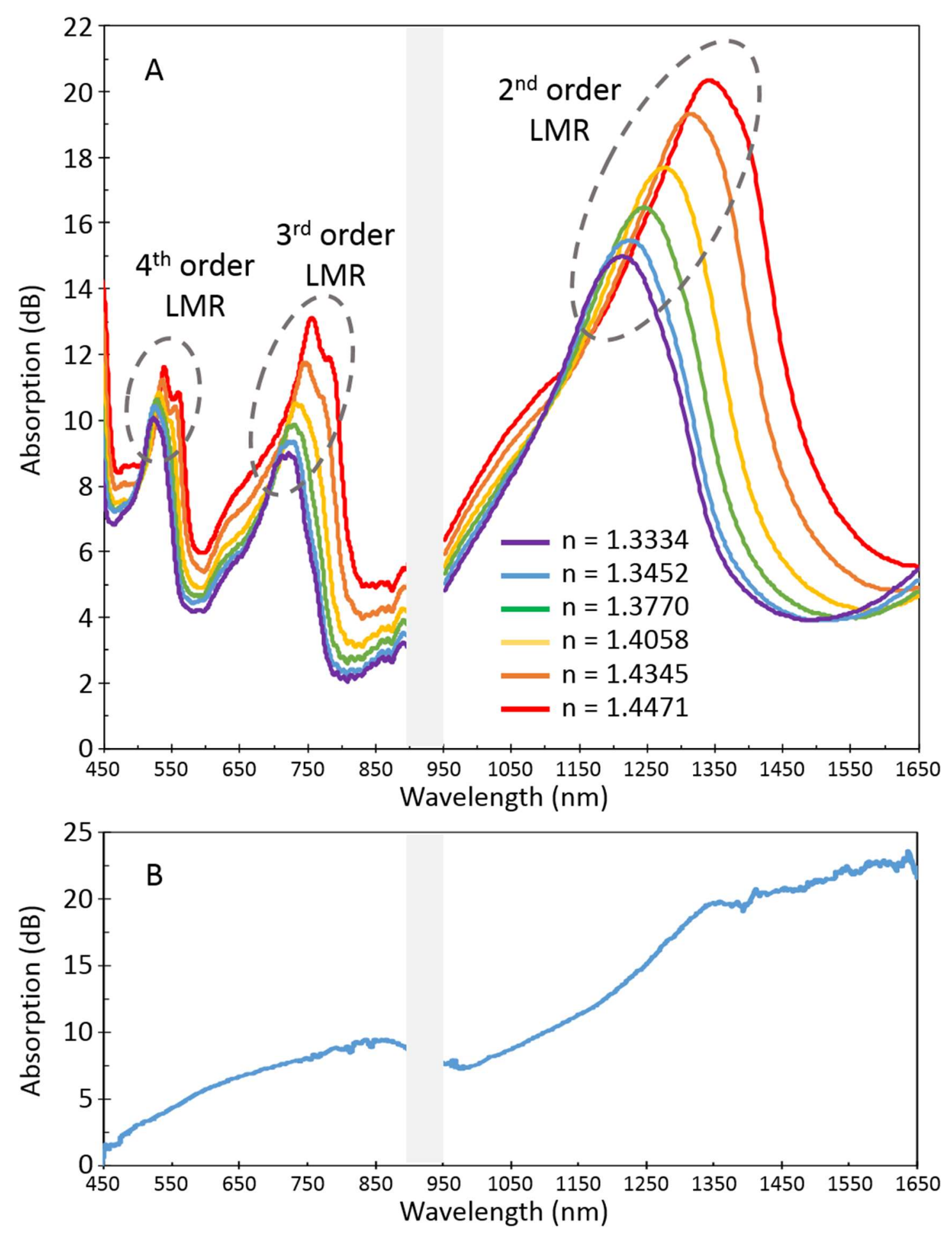

Figure 3: A: Absorption spectra of the sensor surrounded by solutions of different refractive index. The left side of the graph (USB2000) shows two different resonances, one around $500 \mathrm{~nm}$ corresponding to a 4th order LMR and the second one around $750 \mathrm{~nm}$ corresponding to a $3 \mathrm{rd}$ order LMR. On the right side (NIR512), a single resonance of second order can be observed. B: Example spectra of the first order LMR obtained at 2.5 minutes during the deposition process. The two components of the resonance can be observed but the resulting LMR is wider than the spectral window of each spectrometer, making it difficult to work with.

From the spectra shown in fig. $3 a$ it is possible to represent the position of the absorption peaks for each value of external refractive index, and obtain the response of the device as a refractometer. Using a simple algorithm in Matlab we can model the curves as parabolas and 
systematically determine the central peak for each LMR. Fig. 4 shows the sensitivity curves of the $2^{\text {nd }}, 3^{\text {rd }}$ and $4^{\text {th }}$ order LMRs, which have an average sensitivity of $1153.6 \mathrm{~nm} / \mathrm{RIU}, 326.6$ $\mathrm{nm} / \mathrm{RIU}$ and $134.3 \mathrm{~nm} / \mathrm{RIU}$ respectively. These figures agree with the usual behavior of LMRs, as their sensitivity is usually lower for those LMRs of higher order. One important point to observe here is that the sensitivity is not constant and it also increases when the resonance is located at greater wavelengths. Taking this into account, we can see that the $4^{\text {th }}$ order LMR has a maximum sensitivity of $251 \mathrm{~nm} / \mathrm{RIU}$, the $3^{\text {rd }}$ order LMR has a maximum sensitivity of $718 \mathrm{~nm} / \mathrm{RIU}$ and the second order LMR has a sensitivity of up to $2280 \mathrm{~nm} / \mathrm{RIU}$. The sensitivity to $\mathrm{pH}$ variation was also studied. It was determined that being the RI sensitivity over $1153.6 \mathrm{~nm} / \mathrm{RIU}$, sensitivity to $\mathrm{pH}$ is in the range of $0.77 \mathrm{~nm} / \mathrm{pH}$ unit, not having a great impact in these measurements when $\mathrm{pH}$ variation is minimal.

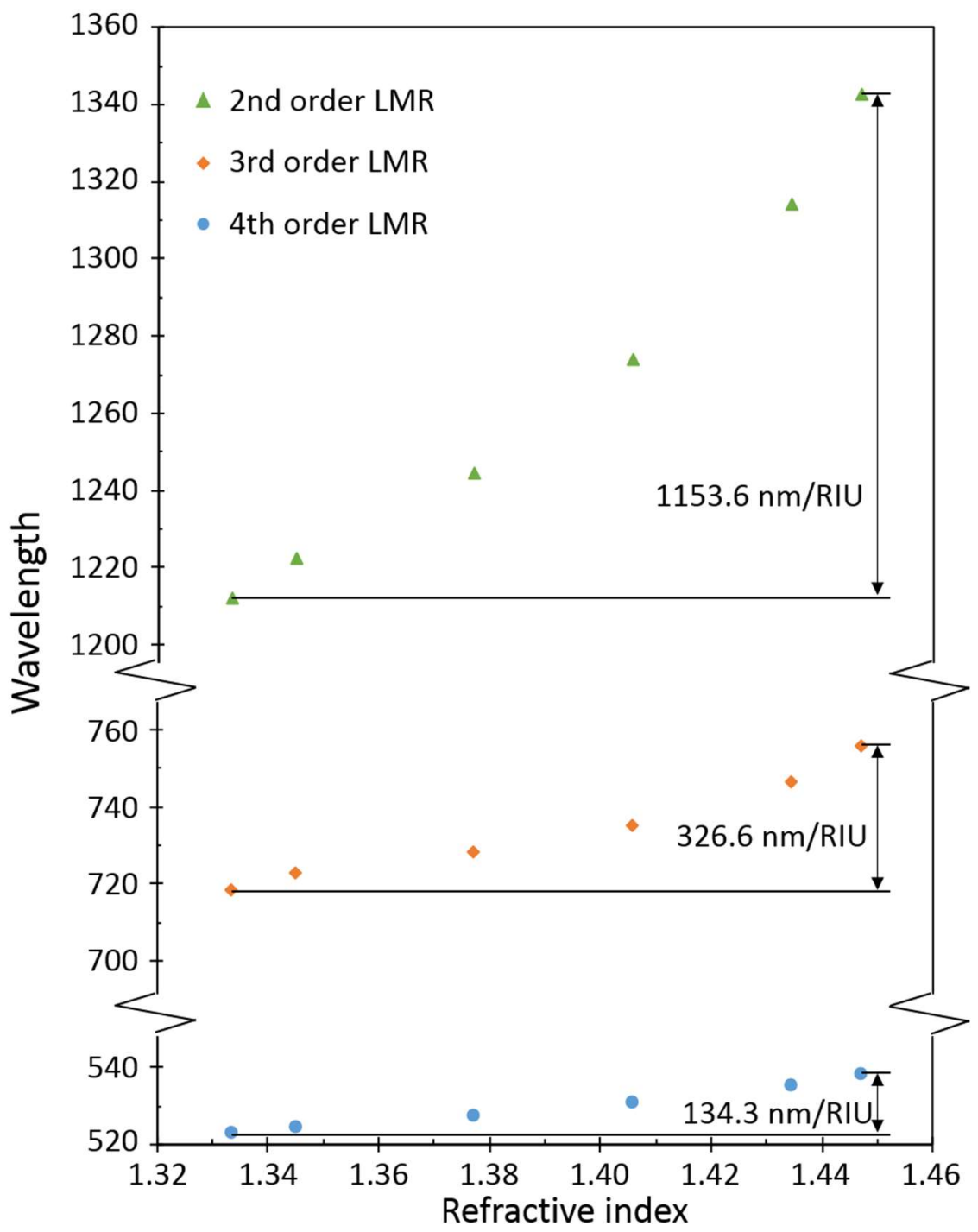

Figure 4: Sensitivity curves of the resonances observed. It can be seen that the wavelength shift is greater for the resonances of lower order.

The sensitivity achieved here could be improved by means of a thinner AZO coating that enables to tune the $1^{\text {st }}$ order LMR in the working spectral window. This could easily be done by adjusting the deposition time, that enables to control the coting thickness and therefore, the resonance 
wavelength position. However, as it was mentioned at the beginning of this section, the observation of the $1^{\text {st }}$ order LMR is difficult using a simple setup like the one presented here because this resonance is too wide, and the separation between both TE and TM components, which are located at different positions, magnifies this effect (fig. 3b).

Alternatively, we propose another method of measurement, using the $2^{\text {nd }}$ order LMR. For this purpose, we have fabricated a second device with a thinner coating $(90 \mathrm{~nm})$ that permits to locate the $2^{\text {nd }}$ order LMR in the visible light spectral region. According to this, a shift on the resonance would produce a change in the color of the transmitted light.

This second device was fabricated following the same procedure as described above with a sputtering fabrication time of 7 minutes. This AZO coating produces a $2^{\text {nd }}$ order LMR at $450 \mathrm{~nm}$ when is surrounded by air and around $490 \mathrm{~nm}$ when surrounded by water. This resonance was chosen as a trade-off: as it was explained above, lower order resonances show more sensitivity, but are also wider, which can make the sensor not color-selective enough for this purpose. In contrast, resonances of higher order, would be more selective, but not sensitive enough. Then, the spectral response of this new device when it is submerged in solutions of increasing refractive index is plotted in fig. 5. This LMR shifts to higher wavelengths, as it also happened with the first device (see fig. 4).

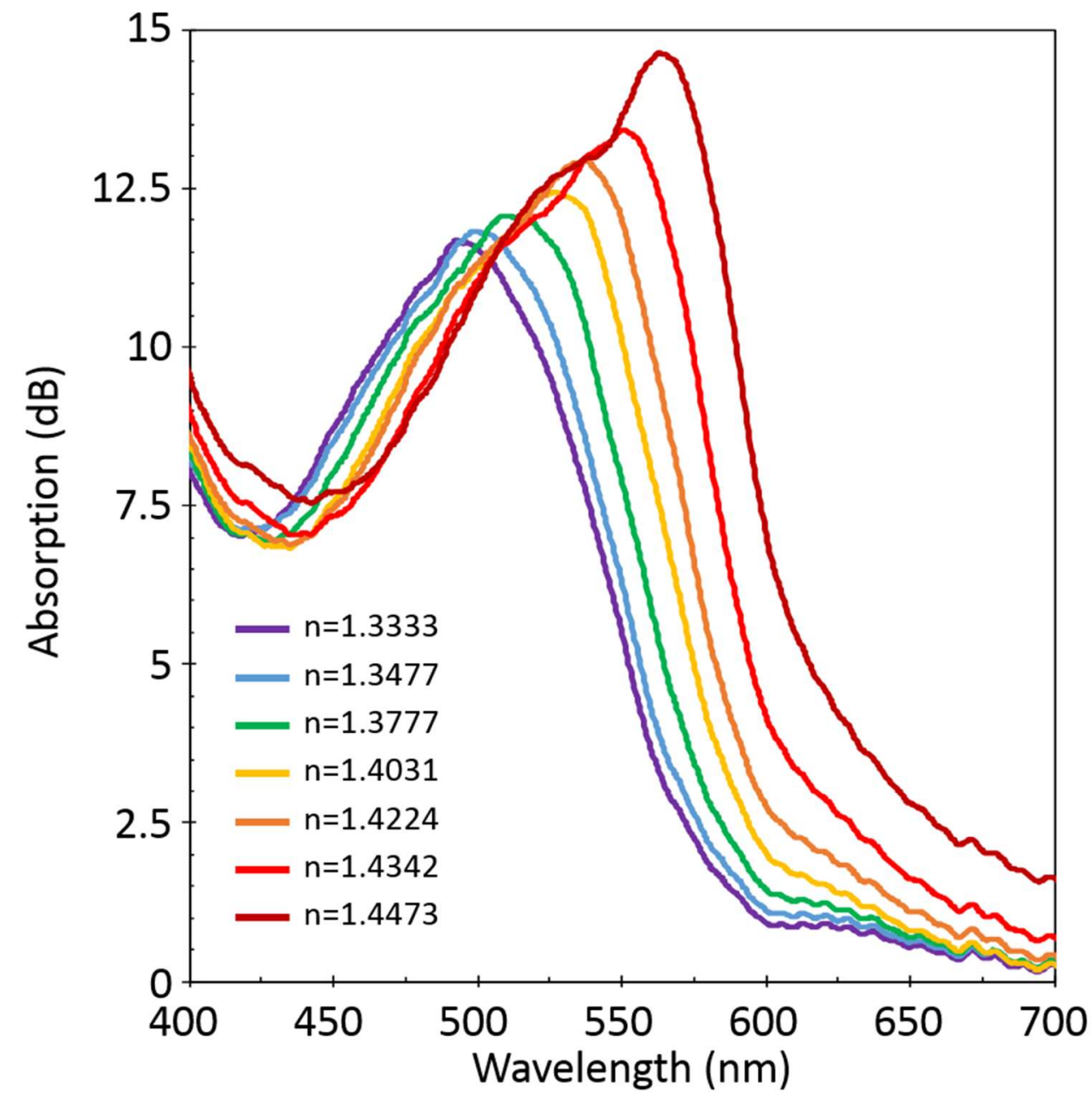

Figure 5: Absorption spectra of the second sensor. It shows a resonance of second order in the visible light range and a red-shift induced when the refractive index of the surrounding media increases. 
In fig. 6 we can also see the representation of the position of each LMR wavelength depending on the refractive index of the medium surrounding the sensitive region. In this case the device shows an average sensitivity of $592 \mathrm{~nm} / \mathrm{RIU}$ in the working range. It is important to note that the sensitivity is not linear as it was mentioned with the previous studied devices and when the refractive index increases, the sensitivity raises too. In particular, in the most sensitive region, this sensor reaches a value of $1142 \mathrm{~nm} / \mathrm{RIU}$.

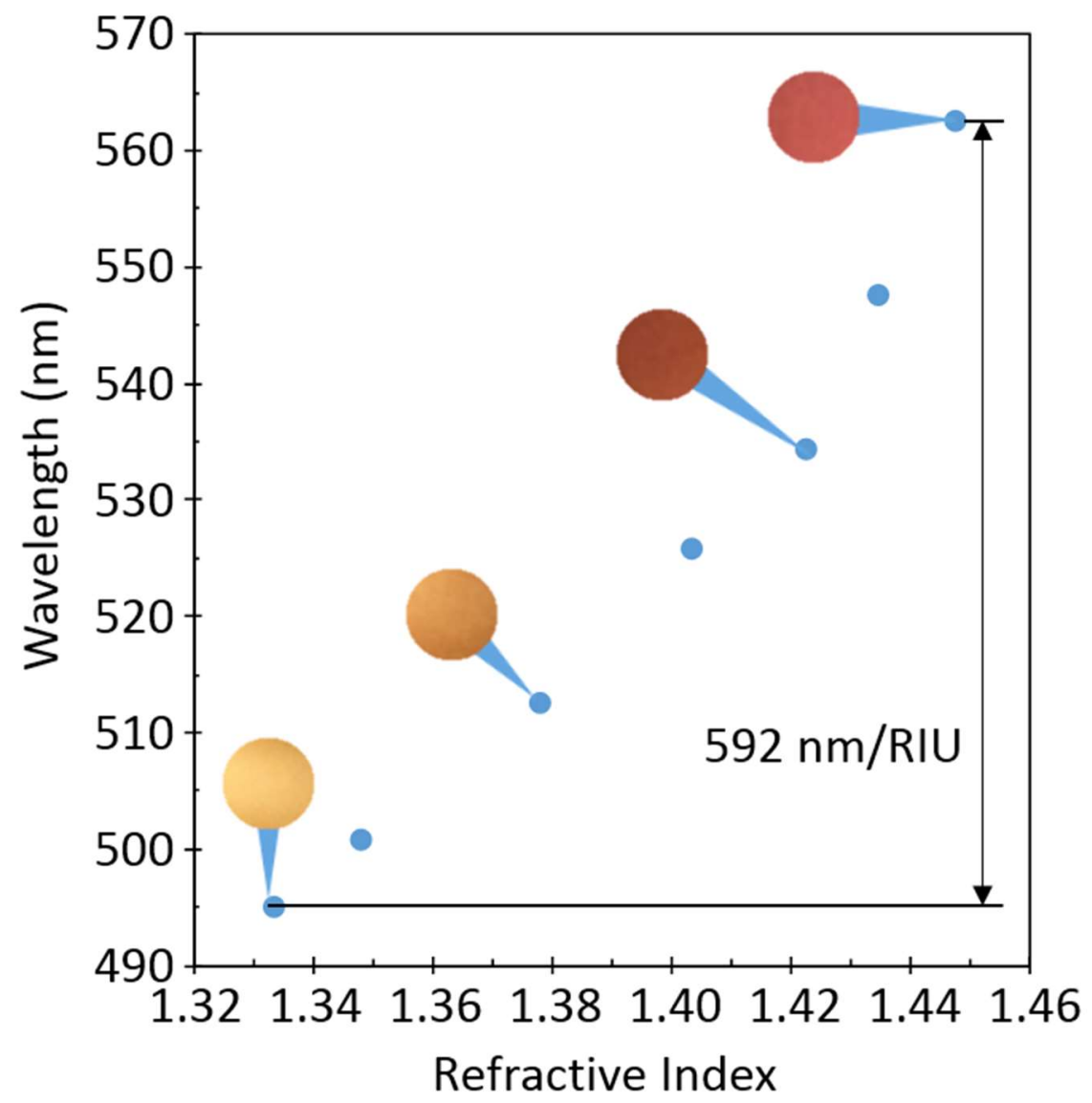

Figure 6: Sensitivity curve of the second order LMR tuned in the VIS range. It has a peak sensitivity of $1142 \mathrm{~nm} / \mathrm{RIU}$. The graph also shows the evolution of the color of the light transmitted through the fiber. The color changes from light yellow to dark red.

This value is lower than in the previous case. Although in both sensors we can study the second order LMR, in the last case, the resonance is located at a lower wavelength, which causes the lower sensitivity. This relation between the sensitivity and the location of the resonance and thickness of the coating has already been studied previously, proving the sensitivity linear dependence of the coating thickness [8]. While the lower sensitivity may represent an inconvenience, the possibility to use this sensor by observing a simple change of color puts it in high value.

As it is observed above, this sensor has one single, relatively narrow resonance in the visible light range. The FWHM of this LMR varies between 76.1 and $81.6 \mathrm{~nm}$. As a result, this resonance changes the color of the light observed at the output of the fiber. Hence, a color variation can be directly associated with a resonance wavelength shift and therefore with a change in the SMRI. As an example, in fig. 6, we can see how the light at the end of the fiber is pale yellow when the sensitive region is in air, but it turns to light orange and then a dark red when the SMRI 
increases. This method presents some limitations, as it is not possible to determine the refractive index with the precision obtained using a spectrometer. However, this change of color visible to the naked eye brings the possibility of working with a much simpler, quicker and economic setup, thus, simplifying the future applications of this device.

\section{Conclusion}

The possibility to design LMR based optical fiber sensors using AZO as a supporting material has been experimentally proven for the first time. Two different sensors have been designed and fabricated. The refractometric response of several LMRs has been observed and characterized, obtaining sensitivities compared to the theoretical values calculated in previous works of other authors. A maximum sensitivity of $2280 \mathrm{~nm} / \mathrm{RIU}$ has been obtained using a second order resonance and a simple setup. Higher sensitivities could still be obtained at the expenses of a more sophisticated setup, for instances, using D-shape optical fiber and polarized light which will allow the separation of TE and TM modes and the characterization of the first order resonance. These refractometers could be the basis for the fabrication of different kind of sensors for industrial as well as biomedical applications.

A different approach for the use of LMR based sensors has also been studied. A simpler setup based on the difference in color of the light induced by the resonance when there is a SMRI variation could be a great economic alternative when only a rough estimation is needed, since only the fiber and a light source would be required. The introduction of AZO as new material for the design of LMR based sensors and the study of its deposition techniques may lead to the development of more applications thanks to its lower cost and its intrinsic properties.

\section{Acknowledgement}

This work was partly supported by the Spanish Agencia Estatal de Investigación (AEI) and Fondo Europeo de Desarrollo Regional (FEDER) (TEC2016-79367-C2-2-R, TEC2016-78047-R). The authors would also like to thanks the Spanish Ministry of Education (FPU15/05663 grant) and the Government of Navarra 0C023/024 BIOPTSENS and 64/2015 research funds. Dafne A. PiñaAzamar thanks CONACYT for scholarship assigned and PFCE-UAT 2016 project for the financial support to carry out this work

\section{References}

1. I. Del Villar, F. J. Arregui, C. R. Zamarreño, J. M. Corres, C. Bariain, J. Goicoechea, C. Elosua, M. Hernaez, P. J. Rivero, A. B. Socorro, A. Urrutia, P. Sanchez, P. Zubiate, D. Lopez, N. De Acha, J. Ascorbe, and I. R. Matias, "Optical sensors based on lossy-mode resonances," Sensors and Actuators B: Chemical 240, 174-185 (2017).

2. M. Piliarik and J. Homola, "Surface plasmon resonance (SPR) sensors: approaching their limits?" Opt. Express, OE 17, 16505-16517 (2009).

3. B. Lee, S. Roh, and J. Park, "Current status of micro- and nano-structured optical fiber sensors," Optical Fiber Technology 15, 209-221 (2009).

4. A. Barrias, J. R. Casas, and S. Villalba, "A Review of Distributed Optical Fiber Sensors for Civil Engineering Applications," Sensors (Basel, Switzerland) 16, 748 (2016). 
5. P. Roriz, O. Frazão, A. B. Lobo-Ribeiro, J. L. Santos, and J. A. Simões, "Review of fiber-optic pressure sensors for biomedical and biomechanical applications," Journal of Biomedical Optics 18, 050903 (2013).

6. N. Paliwal and J. John, "Lossy Mode Resonance (LMR) Based Fiber Optic Sensors: A Review," IEEE Sensors Journal 15, 5361-5371 (2015).

7. A. Ozcariz, C. R. Zamarreño, P. Zubiate, and F. J. Arregui, "Is there a frontier in sensitivity with Lossy mode resonance (LMR) based refractometers?" Scientific Reports 7, 10280 (2017).

8. I. D. Villar, M. Hernaez, C. R. Zamarreño, P. Sánchez, C. Fernández-Valdivielso, F. J. Arregui, and I. R. Matias, "Design rules for lossy mode resonance based sensors," Appl. Opt. , AO 51, 4298-4307 (2012).

9. I. Del Villar, C. R. Zamarreño, M. Hernaez, F. J. Arregui, and I. R. Matias, "Lossy mode resonance generation with indium-tin-oxide-coated optical fibers for sensing applications," Journal of Lightwave Technology 28, 111-117 (2010).

10. C. R. Zamarreño, M. Hernaez, I. Del Villar, I. R. Matias, and F. J. Arregui, "Sensing properties of ITO coated optical fibers to diverse VOCs," Procedia Engineering 5, 653-656 (2010).

11. P. Zubiate, C. R. Zamarreño, P. Sánchez, I. R. Matias, and F. J. Arregui, "High sensitive and selective C-reactive protein detection by means of lossy mode resonance based optical fiber devices," Biosensors and Bioelectronics 93, 176-181 (2017).

12. C. R. Zamarreño, M. Hernáez, I. Del Villar, I. R. Matías, and F. J. Arregui, "Optical fiber pH sensor based on lossy-mode resonances by means of thin polymeric coatings," Sensors and Actuators B: Chemical 155, 290-297 (2011).

13. H. Shen, H. Zhang, L. Lu, F. Jiang, and C. Yang, "Preparation and properties of AZO thin films on different substrates," Progress in Natural Science: Materials International 20, 44-48 (2010).

14. C. Hong, H. Park, J. Moon, and H. Park, "Effect of metal (Al, Ga, and In)-dopants and/or Agnanoparticles on the optical and electrical properties of $\mathrm{ZnO}$ thin films," Thin Solid Films 515, 957-960 (2006).

15. S. J. Henley, M. N. R. Ashfold, and D. Cherns, "The growth of transparent conducting ZnO films by pulsed laser ablation," Surface and Coatings Technology 177-178, 271-276 (2004).

16. N. Paliwal and J. John, "Sensitivity Enhancement of Aluminium Doped Zinc Oxide (AZO) Coated Lossy Mode Resonance (LMR) Fiber Optic Sensors Using Additional Layer of Oxides," in Anonymous (Optical Society of America, 2014/10/19), pp. JTu3A.40.

17. N. Paliwal and J. John, "Theoretical modeling and investigations of AZO coated LMR based fiber optic tapered tip sensor utilizing an additional TiO2 layer for sensitivity enhancement," Sensors and Actuators B: Chemical 238, 1-8 (2017).

18. T. Minami, "Present status of transparent conducting oxide thin-film development for Indium-Tin-Oxide (ITO) substitutes," Thin Solid Films 516, 5822-5828 (2008).

19. J. F. Chang, H. H. Kuo, I. C. Leu, and M. H. Hon, "The effects of thickness and operation temperature on ZnO:Al thin film CO gas sensor," Sensors \& Actuators: B. Chemical 84, 258-264 (2002). 
20. C. Chi, H. Chen, W. Chen, C. Chang, and W. Liu, "Formaldehyde sensing characteristics of an aluminum-doped zinc oxide (AZO) thin-film-based sensor," Sensors \& Actuators: B. Chemical 255, 3017-3024 (2018).

21. W. Tsai, K. Lin, S. Yang, Y. Tsao, and P. Ho, "Fiber-optic surface plasmon resonance-based sensor with AZO/Au bilayered sensing layer," Chin. Opt. Lett. , COL 12, 042801 (2014).

22. C. R. Zamarreño, P. Zubiate, M. Sagües, I. R. Matias, and F. J. Arregui, "Experimental demonstration of lossy mode resonance generation for transverse-magnetic and transverseelectric polarizations," Opt. Lett. , OL 38, 2481-2483 (2013). 